\title{
Management of patients undergoing same-day discharge primary total hip and knee arthroplasty
}

\author{
Andrew Bodrogi MD, Geoffrey F. Dervin MD MSc, Paul E. Beaulé MD
}

Cite as: CMAJ 2020 January 13;192:E34-9. doi: 10.1503/cmaj.190182

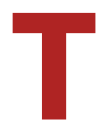
otal hip arthroplasty and total knee arthroplasty are established procedures for the effective treatment of complications of advanced arthritides or avascular necrosis. With new surgical techniques and approaches to perioperative care, the average length of an inpatient stay associated with these procedures has decreased in recent years. ${ }^{1}$ The demand for primary total hip and total knee arthroplasty in patients younger than 65 years has been projected to increase by more than $50 \%$ by $2030 .{ }^{2}$ With the annual frequency of these procedures already rapidly increasing owing to an aging population, same-day discharge arthroplasty may be a feasible solution to the increased pressure to decrease health care costs. ${ }^{3}$

We review observational and interventional research on the effectiveness of same-day discharge total hip and total knee arthroplasty (Box 1). In this review, same-day discharge arthroplasty refers to protocols that see patients discharged home on the same calendar day as the procedure, usually within 4 to 8 hours after the end of the surgery. This is distinct from discharge within 23 hours after surgery, which is often labelled as "outpatient" in the literature. However, many large database studies do not distinguish between the 2 kinds of protocols, and some evidence presented herein includes patients who spent up to 23 hours in hospital.

\section{Which patients are candidates for outpatient total hip and total knee arthroplasty?}

Many factors must be considered when selecting patients for same-day discharge total joint arthroplasty. Ensuring patient safety is important. An analysis of American College of Surgeons National Surgical Quality Improvement Program data identified patient age older than 80 years as an independent predictor for adverse events or readmission in this population (odds ratio of 4.16). ${ }^{4}$ As such, many protocols and studies have used this cut-off in their selection criteria, and most patients selected for sameday procedures are relatively young - in their $50 \mathrm{~s}$ and $60 \mathrm{~s} .{ }^{5-8}$ Other commonly used criteria include a Timed Up and Go test of 10 seconds or less, ${ }^{9}$ and an American Society of Anesthesiologists classification of 1-2, measured preoperatively. ${ }^{4,10}$

Exclusion criteria focus primarily on the presence of certain comorbidities. Observational studies have shown that, among candidates for knee and hip arthroplasty, bleeding disorders, liver cirrhosis and stage 3-5 renal disease are independent risk factors for

\section{KEY POINTS}

- Same-day discharge total hip and knee arthroplasty is becoming more common, made feasible by perioperative advances such as minimally invasive surgical approaches, the use of tranexamic acid and multimodal and pre-emptive analgesia.

- According to protocols developed to ensure patient safety, candidates for same-day surgery are people younger than 80 years without preoperative bleeding disorders, cirrhosis, clinically important cardiac disease or end-stage renal disease.

- When patients are selected appropriately, rates of adverse events and functional outcomes are similar to those observed among patients who undergo inpatient-protocol arthroplasty, patient satisfaction is high, and procedures are cost-effective.

- Careful education of patients, by surgeons and primary care physicians, can help to dispel myths about outpatient total joint arthroplasty and thereby optimize success.

adverse events or readmission (Box 2)., ${ }^{411-21}$ Findings regarding the contribution of elevated body mass index ( $>35$ or $40 \mathrm{~kg} / \mathrm{m}^{2}$ ) and diabetes mellitus to an increase in adverse events are mixed. ${ }^{4,15,16} \mathrm{~A}$ retrospective observational study of more than 1000 patients found that most complications $(71 \%)$ requiring physician intervention after total hip or total knee arthroplasty were cardiopulmonary in nature. ${ }^{14}$ However, cardiac history (myocardial infarction, previous cardiac surgery or stents) alone does not seem to increase risk of adverse events in otherwise well-selected patients when intraoperative blood loss is minimized and perioperative hypotension is carefully managed. ${ }^{6,8,16-18}$ Similarly, history of chronic obstructive pulmonary disease ${ }^{17-20}$ and previous deep vein thrombosis or pulmonary embolus ${ }^{6,8,18}$ have not been clearly associated with increased rates of adverse events.

Social factors also play a role in the appropriate selection of patients. Many protocols involve some form of preoperative counselling outlining the general flow of the hospital stay, pain management and postoperative physical therapy, whether it be from a surgeon, nurse, coordinator or physiotherapist. ${ }^{6-8}$ Patients must have not only the mental capacity to participate actively in these sessions, but also a means of transportation in order to attend. The literature has identified a strong social support system as essential for assistance (both physically and mentally) in the perioperative period, particularly the first night after discharge. ${ }^{21}$ 


\section{Box 1: Summary of literature review}

We performed a systematic literature search of PubMed (1980 to December 2018) and MEDLINE (1950 to December 2018) databases to identify eligible studies on same-day discharge total hip arthroplasty and total knee arthroplasty. We used a combination of subject headings, including "total joint arthroplasty," "hip arthroplasty," "knee arthroplasty," "outpatient," "same-day discharge," "length of stay," "ambulatory," "rapid recovery protocol," "short stay," and "daycase" and limited the search to articles written in English. Reference lists of selected articles were also reviewed for additional studies. Relevant articles included clinical trials, meta-analyses, prognostic studies, observational studies and retrospective studies.

Several risk assessment scores have been developed to assist with selection of patients for same-day discharge total joint arthroplasty to minimize risk of adverse events or readmission. ${ }^{22,23}$ The Outpatient Arthroplasty Risk Assessment score considers the presence, severity and medical optimization of various conditions and assigns a score in 9 comorbidity areas, including general medical, hematological and cardiac. A cumulative score of less than 60 more precisely predicted same-day or next-day discharge than American Society of Anesthesiologists score or Charlson comorbidity index when tested in a retrospective cohort of 1120 patients. ${ }^{22}$ The Readmission Risk Assessment Tool assigns points for 9 modifiable risk factors, such as Staphylococcus aureus nasal colonization, tobacco use, obesity, cardiovascular disease and diabetes. A score of 3 or greater was associated with higher odds of readmission after primary hip or knee arthroplasty when tested in a large retrospective cohort. ${ }^{23}$ This tool may therefore also be useful when selecting lower-risk patients for outpatient arthroplasty.

\section{What perioperative advances have enabled same-day discharge total joint arthroplasty?}

The most common barriers to discharge in patients undergoing total joint arthroplasty are hypotension, pain and nausea. ${ }^{24} \mathrm{How}-$ ever, greater uptake of minimally invasive surgical approaches has allowed patients to benefit from decreased soft-tissue damage, reduced blood loss, less postoperative pain and quicker mobilization after surgery. ${ }^{25,26}$ Many of the studies that have assessed the feasibility and effectiveness of same-day discharge have used either the anterior approach,,$^{5,27,28}$ a 2-incision technique, ${ }^{17,29}$ or minimally invasive posterior ${ }^{18}$ approaches for total hip arthroplasties, and quadriceps-sparing approaches ${ }^{6,19}$ for total knee arthroplasties. Although use of minimally invasive approaches is important, perioperative innovations in managing blood loss and optimizing analgesia have contributed most substantially to making same-day discharge arthroplasties possible.

A vital advance in minimizing perioperative blood loss has been the routine use of tranexamic acid, an antifibrinolytic agent, often given before surgical incision, which binds to plasminogen and prevents fibrin degradation. ${ }^{30}$ Recent metaanalyses have shown strong evidence that tranexamic acid, given

\section{Box 2: Outpatient total joint arthroplasty exclusion criteria $^{4,9-21}$}

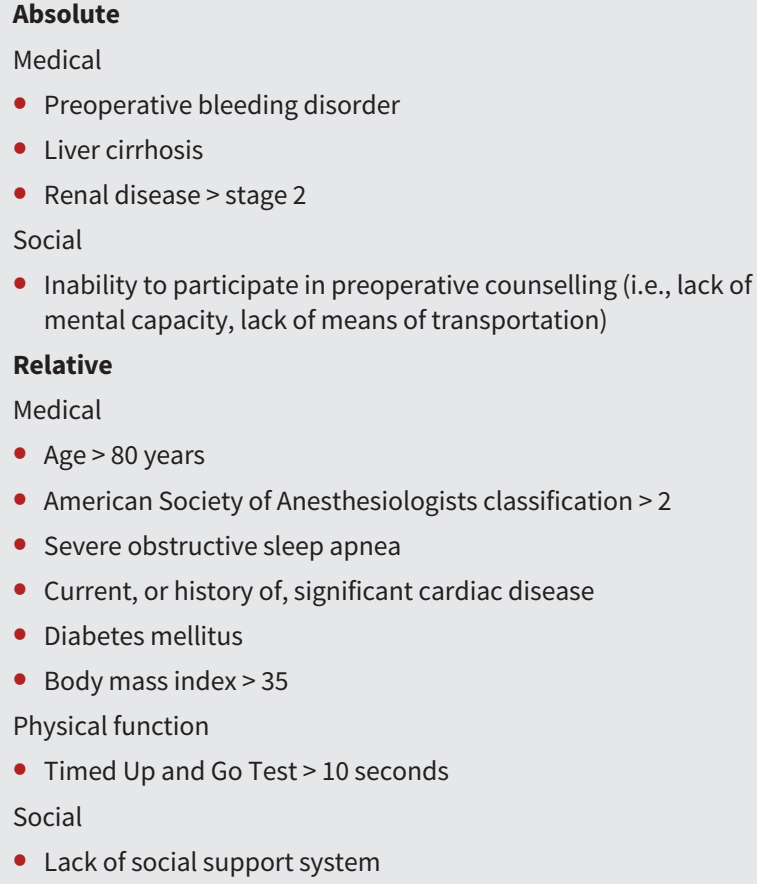

- Lack of social support system

topically, orally or intravenously, substantially decreases blood loss in both total hip and total knee arthroplasty, with an associated decreased risk of transfusion. ${ }^{31,32}$

Successful perioperative pain management for total joint arthroplasty is multimodal, ${ }^{33,34}$ with pre-emptive treatment to prevent central sensitization of the nervous system, ${ }^{6,34,35}$ limited use of narcotics (particularly intravenous) to prevent acute adverse effects that include hypotension or oversedation, and to minimize nausea and urinary retention, 2 barriers to delayed patient discharge. ${ }^{17}$ Local infiltrative analgesia - usually periarticular injection of a dilute cocktail that commonly comprises ropivacaine, epinephrine and ketorolac tromethamine - is a commonly used adjunct. ${ }^{36-38}$ Both general and neuraxial anesthetics have been used for same-day discharge arthroplasty procedures. ${ }^{5,17,19,28}$ Although national database studies have shown higher rates of complications with general anesthesia in populations of patients undergoing total joint arthroplasty as a whole, ${ }^{39,40}$ higher complication rates have yet to be shown among those undergoing outpatient procedures, presumably as a result of selecting healthier patients. Because a meta-analysis of studies of neuraxial anesthesia that included intrathecal morphine showed an increased risk of nausea, vomiting and pruritis, ${ }^{41}$ newer protocols for neuraxial anesthesia avoid use of intrathecal opioids. The findings of a recent matched cohort analysis also suggest that higher doses of intrathecal local anesthetic (such as bupivacaine) may delay recovery of function of the lower extremities, thereby also delaying safe discharge. ${ }^{5}$

Use of postoperative cryotherapy to augment postoperative pain management and rehabilitation in patients undergoing total joint arthroplasty has increased recently, although a 2012 
Cochrane review found low-quality evidence for improved range of motion, and very low-quality evidence for a small decrease in blood loss and pain scores only at 48 hours, with no evidence to support change in transfusion rate or analgesia use, from cryotherapy after total knee arthroplasty.42 A more recent systematic review of randomized controlled trials of cryotherapy after total hip arthroplasty showed no significant difference for blood loss or pain management in the first 24 hours. ${ }^{43}$

For total knee arthroplasty specifically, use of a tourniquet during surgery has been shown to decrease intraoperative blood loss, although a meta-analysis found overall calculated blood loss and need for transfusion to be unaffected by intraoperative tourniquet use. ${ }^{44}$ Although visualization of the surgical field may improve with use of a tourniquet, it is important to consider potential adverse effects, which include decreased range of motion in the first days postoperatively and increased risk of thrombotic events, hematoma and nerve palsy. ${ }^{44}$ Some studies have also shown increased postoperative pain with tourniquet use $\mathrm{u}^{45,46}$ as well as increased quadriceps dysfunction. ${ }^{47,48}$ Whether to use no tourniquet, to limit use or to use one for the full procedure is a question that remains unanswered for same-day discharge joint arthroplasty.

Single-shot or continuous femoral nerve blocks have long been used effectively to decrease postoperative pain in patients undergoing total knee arthroplasty both on their own or to complement neuraxial anesthesia. ${ }^{49}$ Some centres have used femoral nerve blocks instead of general or neuraxial anesthesia among patients undergoing same-day procedures with success. ${ }^{7,20}$ However, an adverse effect of this approach is acute quadriceps weakness with decreased mobility and increased risk of falls, ${ }^{50,51}$ which means that femoral nerve block is unlikely to be useful in same-day discharge total knee arthroplasty protocols. Adductor canal block, which involves injection of local anesthetic into the adductor canal, anesthetizing the posterior branch of the obturator nerve and branches of the femoral nerve distal to the quadriceps motor branches, ${ }^{52}$ was shown in a recent meta-analysis to have equivalent pain control to femoral nerve block, with better preservation of quadriceps strength and improved mobilization..$^{53}$ Another study noted subjectively lower worst pain, and greater pain relief at 24 hours, when adductor canal block was combined with periarticular infiltration compared with periarticular infiltration alone. ${ }^{54}$ As more anesthesiologists become familiar with this technique, its widespread use as an analgesic adjunct could decrease opioid requirements and may allow more patients to be eligible for sameday discharge total knee arthroplasty.

\section{What can a patient expect after outpatient total knee or total hip arthroplasty?}

A typical protocol, as described in a recent study, is as follows. ${ }^{5}$ Patients are kept in the postanesthesia care unit for observation until they meet discharge criteria, which are usually based on a postanesthetic recovery score that accounts for the level of consciousness, blood pressure, heart rate, respiratory rate, oxygen levels, nausea, pain levels and recovery of lower limb muscle function. Once cleared to leave postanesthesia recovery, patients return to the surgical day care unit to be assessed by a physiotherapist.
Patients are cleared for discharge only if they can effectively and safely perform independent transfers and climb stairs with an assistive device (such as crutches or a walker). Repeat administrations of intravenous antibiotics and oral tranexamic acid are given before discharge. An anteroposterior pelvic radiograph for total hip arthroplasty, and anteroposterior pelvic and lateral knee radiographs for total knee arthroplasty, are performed and reviewed by the surgical team. If the postoperative radiograph is found to be satisfactory, patients are then discharged only if they exhibit stable vital signs, can tolerate a regular diet, have adequate pain control with oral analgesics and feel comfortable being discharged home. Postoperative stays usually range from 4 to 8 hours.

Although same-day discharge arthroplasty protocols differ in some elements, safety of discharge is their common theme. Most protocols have specific physical therapy milestones that must be met before a patient can be discharged, usually involving independent transfer from supine to chair to stand, ambulation for a set distance using an assistive device, and the ability to ascend and descend a flight of stairs..$^{6-8,17-19,28}$ Protocols must also involve prophylactic therapies for hypovolemia, pain and nausea, 5,17 and treatments to address poorly controlled pain, nausea, hypotension, dizziness or oversedation. ${ }^{17}$ Although success has been reported at an ambulatory surgical centre, ${ }^{20}$ any new protocol should ideally be piloted at a centre with inpatient capabilities, to allow for admission if necessary. ${ }^{6,28,55}$

Lastly, postdischarge supports and surveillance vary greatly, ranging from merely the ability to call the clinic or surgeon's administrative assistant if there are issues, to remote postoperative home monitoring systems that wirelessly transmit vital signs and pain scores in real time. ${ }^{56,57}$ Usual postoperative wound checks are appropriate. Studies that have compared patients treated using standard inpatient protocols with those treated under an outpatient protocol have shown no difference in the rate of superficial or deep surgical infections. ${ }^{7,28}$

\section{How do outcomes of outpatient protocols compare with those of traditional inpatient protocols?}

One multicentre, prospective, randomized trial with a total of 220 patients undergoing total hip arthroplasty compared the inpatient with the outpatient approach and found no difference in re-operations, hospital readmissions, emergency department visits or acute office visits. ${ }^{28} \mathrm{~A}$ recent matched cohort study performed at a Canadian centre showed no difference in the rate or severity of adverse events up to 90 days between same-day discharge and inpatient unilateral total hip arthroplasties. In the cohort of 136 patients who were discharged on the day of surgery, there were no serious adverse events. ${ }^{5}$ Other matched cohort studies comparing same-day discharge with inpatient total joint arthroplasty have shown no difference in complications, readmissions or emergency department visits, ${ }^{27,58}$ with 1 study even showing a significantly lower rate of 90-day readmission rates in the same-day discharge cohort. ${ }^{59}$ However, there are relatively few high-quality comparative studies.

The findings of studies that did not distinguish between sameday discharge and discharge within 23 hours can still provide 
some important insights. A recent systematic review with inclusion of 10 publications (a mix of same-day discharge and discharge within $23 \mathrm{~h}$ ) with a total of 1009 patients, of which $94.5 \%$ were successfully discharged on the same day as surgery, showed the reoperation rate was $1.98 \%$. The rate of readmission within 90 days was $0.89 \%$, whereas the rate of minor and major complications were $1.29 \%$ and $0.10 \%$, respectively. ${ }^{24}$ Similarly, a review of National Surgical Quality Improvement Program data of patients discharged within 24 hours postsurgery showed rates of severe adverse events and unplanned readmissions of $1.3 \%$ and $1.9 \%$, respectively. ${ }^{4}$ These results compare favourably with general US database rates, which range from $1.7 \%$ to $6.4 \%$ for 30 -day readmission rates and from $4.0 \%$ to $11.5 \%$ for 30 -day complication rates. ${ }^{60-62}$ These rates are for all arthroplasty patients (of which a very small percentage would be outpatient programs; about 1\%-2\%). Furthermore, using National Surgical Quality Improvement Program data, matched cohort studies of within23-hours discharge versus inpatient total joint arthroplasty showed no difference in overall adverse events or readmission rates. ${ }^{15,63}$ Of note, however, patients discharged within 23 hours had a lower risk of thromboembolic events but an increased risk of return to the operating room (particularly in the total knee arthroplasty cohort). However, the reason for reoperation could not be elucidated because of lack of details in the database, and this result has not been demonstrated in other studies. ${ }^{15}$

In terms of functional outcomes, a prospective comparative cohort study of same-day discharge and inpatient total knee arthroplasties showed no difference in Knee Injury and Osteoarthritis Outcome Score and Western Ontario and McMaster Universities Osteoarthritis Index subscores at 1- and 2-year follow-ups. ${ }^{58}$ Quality of recovery scores (QoR-9) were significantly better on postoperative day 1 in the outpatient group, with no significant difference at other time points up to 28 days. ${ }^{58} \mathrm{~A}$ prospective matched cohort study of 64 patients who were discharged within 23 hours after total knee arthroplasty found no difference in Knee Society knee and function scores or knee range of motion at a mean follow-up of 24 months. ${ }^{7}$ With respect to total hip arthroplasty, the previously mentioned prospective randomized trial comparing same-day discharge and inpatient total hip arthroplasty showed no difference in visual analogue scale pain scores or Harris hip score functions at 4 weeks. ${ }^{28}$ Visual analogue scale scores were significantly worse on postoperative day 1 for outpatients by a mean of 0.9 , but this is below the minimally clinically important difference of 1.9 for total hip arthroplasty. ${ }^{64}$

Studies assessing patient satisfaction have indicated that between $80 \%$ and $96 \%$ of patients report that they would have outpatient arthroplasty again. ${ }^{17,18,20}$ Among 52 total hip arthroplasty outpatients, $94 \%$ would recommend the same-day procedure to others, and $87 \%$ felt that having a same-day discharge total hip arthroplasty improved their confidence and accelerated their recovery. ${ }^{18} \mathrm{~A}$ qualitative study using semistructured interviews with patients and their caregivers also found high levels of satisfaction. ${ }^{65}$ The authors identified the following influential factors for patients favouring an outpatient procedure: previous negative experiences in the hospital, fear of hospital-acquired illnesses and a preference for recovering in the comfort and pri- vacy of their own home. Although these initial results are largely positive, $16 \%-19 \%$ of patients reported postoperative pain to be a problem. ${ }^{18,20}$ As outpatient protocols continue to evolve, it will be important to address pain concerns, whether through better patient and caregiver education, improved analgesic pathways or both.

\section{Do outpatient joint arthroplasty programs save the health system money?}

In North America, pressure from third-party payers to decrease health care costs while maintaining quality has driven shorter lengths of stay in hospital, including same-day discharge surgical programs. ${ }^{66}$ One American cost-analysis of same-day discharge versus standard protocol total hip arthroplasty (using data from a single facility that included costs associated with preoperative physiotherapy for outpatient candidates, in-hospital costs, postoperative physiotherapy and home nursing support for outpatient candidates) found that total average cost saving per sameday discharge case was more than $7 \%$, or US\$3105. ${ }^{67}$ This was despite the additional physical therapy and support costs to ensure safety for outpatient candidates. Similar results were shown for outpatient total knee arthroplasty in a study that analyzed Medicare costs, including those attributed to knee osteoarthritis, up to 2 years after surgery..$^{55}$ A prospective case-control study suggest that these fiscal benefits could translate to the Canadian health care system. ${ }^{68}$ The authors found a median cost saving of $32.7 \%$, or $\$ 3155$, for same-day discharge total knee arthroplasty, although this study considered only in-hospital costs and further studies are needed to refine estimates.

Considering that more than 120000 primary total joint arthroplasties are performed annually in Canada, ${ }^{69}$ a wide rollout of same-day discharge total knee and total hip arthroplasty programs could lead to savings of many millions of dollars for the Canadian health system. A recent Canadian study that retrospectively examined 400 cases treated at a single centre concluded that about $55 \%$ of patients who had undergone total knee arthroplasty would have been appropriate candidates for sameday discharge arthroplasty. ${ }^{70}$

Furthermore, increasing the proportion of outpatient arthroplasties in Canada could assist in addressing problems of inpatient bed capacity and associated costs of increased staffing and cancelled elective operative cases. As outlined above, strict patient selection, use of perioperative advances and establishment of appropriate safeguards are essential, as unplanned readmissions are costly. ${ }^{71}$

\section{How can primary care physicians optimize outcomes for patients who undergo same-day discharge total joint arthroplasty?}

Actively addressing modifiable risk factors, such as smoking and poor nutrition, preoperatively or even before referral, can improve outcomes after any joint arthroplasty procedure. In particular, a history of smoking is an independent risk factor for having an adverse event or unplanned readmission after surgery. ${ }^{4,72,73}$ 
Similarly, hypoalbuminemia, a marker of malnutrition, has also been correlated with higher complication and readmission rates for patients undergoing both early-discharge and regular inpatient total joint arthroplasty. ${ }^{4,74,75}$

Good communication is important to successful same-day discharge total joint arthroplasty, not only from the operating surgeon, but also from the primary physician. One study showed that even though most patients are aware of outpatient total joint arthroplasty and believe their home is the ideal place to recover, 54\% still expected a hospital stay of at least 2 days. ${ }^{76} \mathrm{~A}$ change in these patient perceptions could start with good communication at the time of referral by the primary care physician, as entrenched preconceptions can be difficult to change later on. Qualitative research has identified patient concerns unique to same-day discharge total hip arthroplasty regarding expectations for recovery, medications and their impact on mobility, the timing of postoperative education and the availability of formal care. ${ }^{65}$ Preoperative education, proactively addressing concerns, and clarifying the recovery process and pain expectations were suggested to be key to overcoming these challenges. ${ }^{65}$ Although much of the communication is the responsibility of the operating surgeon, having primary care physicians echo evidence-based advice ensures that patients receive consistent information in their multidisciplinary care.

\section{Conclusion}

Through improvements in surgical technique and perioperative care, same-day discharge total joint arthroplasty has become a safe procedure for a subset of patients, with outcomes and patient satisfaction equivalent to standard inpatient protocols. In a health care system straining to maintain fiscal viability, these procedures offer a way for health care costs to be decreased substantially. Protocols continue to evolve. However, patient safety must remain the priority with any change. Careful education of patients, by surgeons and primary care physicians, can help shift any misconceptions about outpatient total joint arthroplasty procedures and thereby optimize success.

\section{References}

1. Stambough JB, Nunley RM, Curry MC, et al. Rapid recovery protocols for primary total hip arthroplasty can safely reduce length of stay without increasing readmissions. J Arthroplasty 2015;30:521-6.

2. Kurtz SM, Lau E, Ong K, et al. Future young patient demand for primary and revision joint replacement: national projections from 2010 to 2030. Clin Orthop Relat Res 2009;467:2606-12.

3. Nwachukwu BU, Hamid KS, Bozic KJ. Measuring value in orthopaedic surgery. JBJS Rev 2013;1: pii: 01874474-201311000-00003.

4. Sher A, Keswani A, Yao DH, et al. Predictors of same-day discharge in primary total joint arthroplasty patients and risk factors for post-discharge complications. J Arthroplasty, 2017;32:S150-S156.e1.

5. Richards M, Alyousif H, Kim JK, et al. An evaluation of the safety and effectiveness of total hip arthroplasty as an outpatient procedure: a matched-cohort analysis. J Arthroplasty 2018;33:3206-10.

6. Berger RA, Kusuma SK, Sanders SA, et al. The feasibility and perioperative complications of outpatient knee arthroplasty. Clin Orthop Relat Res 2009;467:1443-9.

7. Kolisek FR, McGrath MS, Jessup NM, et al. Comparison of outpatient versus inpatient total knee arthroplasty. Clin Orthop Relat Res 2009;467:1438-42.

8. Chen D, Berger RA. Outpatient minimally invasive total hip arthroplasty via a modified Watson-Jones approach: technique and results. Instr Course Lect 2013;62:229-36.
9. Poitras S, Wood KS, Savard J, et al. Predicting early clinical function after hip or knee arthroplasty. Bone Joint Res 2015;4:145-51.

10. Belmont PJ Jr, Goodman GP, Hamilton W, et al. Morbidity and mortality in the thirty-day period following total hip arthroplasty: risk factors and incidence. $J$ Arthroplasty 2014;29:2025-30.

11. Courtney PM, Froimson MI, Meneghini RM, et al. Can total knee arthroplasty be performed safely as an outpatient in the medicare population? J Arthroplasty 2018;33(7S):S28-31.

12. O'Malley NT, Fleming FJ, Gunzler DD, et al. Factors independently associated with complications and length of stay after hip arthroplasty: analysis of the National Surgical Quality Improvement Program. J Arthroplasty 2012;27:1832-7.

13. Warth LC, Pugely AJ, Martin CT, et al. Total joint arthroplasty in patients with chronic renal disease: Is it worth the risk? J Arthroplasty 2015;30(Suppl):51-4.

14. Courtney PM, Rozell JC, Melnic CM, et al. Who should not undergo short stay hip and knee arthroplasty? Risk factors associated with major medical complications following primary total joint arthroplasty. J Arthroplasty 2015;30(Suppl):1-4.

15. Basques BA, Tetreault MW, Della Valle CJ. Same-day discharge compared with inpatient hospitalization following hip and knee arthroplasty. J Bone Joint Surg Am 2017;99:1969-77.

16. Lovald S, Ong K, Lau E, et al. Patient selection in outpatient and short-stay total knee arthroplasty. J Surg Orthop Adv 2014;23:2-8.

17. Berger RA, Sanders SA, Thill ES, et al. Newer anesthesia and rehabilitation protocols enable outpatient hip replacement in selected patients. Clin Orthop Relat Res 2009;467:1424-30.

18. Dorr LD, Thomas DJ, Zhu J, et al. Outpatient total hip arthroplasty. J Arthroplasty 2010;25:501-6.

19. Berger RA, Sanders S, D’Ambrogio E, et al. Minimally invasive quadriceps-sparing TKA: results of a comprehensive pathway for outpatient TKA. J Knee Surg 2006; 19:145-8.

20. Parcells PB, Giacobbe D, Macknet D, et al. Total joint arthroplasty in a stand-alone ambulatory surgical center: short-term outcomes. Orthopedics 2016;39:223-8.

21. Thienpont $\mathrm{E}$, Lavand'homme $\mathrm{P}$, Kehlet $\mathrm{H}$, et al. The constraints on day-case total knee arthroplasty: the fastest fast track. Bone Joint J 2015;97-B(Suppl A):40-4.

22. Meneghini RM, Ziemba-Davis M, Ishmael MK, et al. Safe selection of outpatient joint arthroplasty patients with medical risk stratification: the "Outpatient Arthroplasty Risk Assessment Score”. J Arthroplasty 2017;32:2325-31.

23. Boraiah S, Joo L, Inneh IA, et al. Management of modifiable risk factors prior to primary hip and knee arthroplasty: a readmission risk assessment tool. J Bone Joint Surg Am 2015;97:1921-8.

24. Hoffmann JD, Kusnezov NA, Dunn JC, et al. The shift to same-day outpatient joint arthroplasty: a systematic review. J Arthroplasty 2018;33:1265-74.

25. Khanna A, Gougoulias N, Longo UG, et al. Minimally invasive total knee arthroplasty: a systematic review. Orthop Clin North Am 2009;40:479-89.

26. Cheng T, Feng JG, Liu T, et al. Minimally invasive total hip arthroplasty: a systematic review. Int Orthop 2009;33:1473-81.

27. Aynardi M, Post Z, Ong A, et al. Outpatient surgery as a means of cost reduction in total hip arthroplasty: a case-control study. HSS J 2014;10:252-5.

28. Goyal N, Chen AF, Padgett SE, et al. Otto Aufranc Award: a multicenter, randomized study of outpatient versus inpatient total hip arthroplasty. Clin Orthop Relat Res 2017;475:364-72.

29. Berger RA, Jacobs JJ, Meneghini RM, et al. Rapid rehabilitation and recovery with minimally invasive total hip arthroplasty. Clin Orthop Relat Res 2004(429):239-47.

30. Okamoto S, Sato S, Y, et al. An active stereo-isomer (trans-form) of AMCHA and its antifibrinolytic (antiplasminic) action in vitro and in vivo. Keio J Med 1964; 13:177-85.

31. Fillingham YA, Ramkumar DB, Jevsevar DS, et al. The efficacy of tranexamic acid in total knee arthroplasty: a network meta-analysis. J Arthroplasty 2018;33:3090-98.e1.

32. Fillingham YA, Ramkumar DB, Jevsevar DS, et al. The efficacy of tranexamic acid in total hip arthroplasty: a network meta-analysis. J Arthroplasty 2018;33:3083-89.e4.

33. Parvizi J, Miller AG, Gandhi K. Multimodal pain management after total joint arthroplasty. J Bone Joint Surg Am 2011;93:1075-84.

34. Lee KJ, Min BW, Bae KC, et al. Efficacy of multimodal pain control protocol in the setting of total hip arthroplasty. Clin Orthop Surg 2009;1:155-60.

35. Woolf CJ, Chong MS. Preemptive analgesia-treating postoperative pain by preventing the establishment of central sensitization. Anesth Analg 1993;77:362-79.

36. Busch CA, Shore BJ, Bhandari R, et al. Efficacy of periarticular multimodal drug injection in total knee arthroplasty. A randomized trial. J Bone Joint Surg Am 2006;88:959-63.

37. Kelley TC, Adams MJ, Mulliken BD, et al. Efficacy of multimodal perioperative analgesia protocol with periarticular medication injection in total knee arthroplasty: a randomized, double-blinded study. J Arthroplasty 2013;28:1274-7.

38. Jiang J, Teng Y, Fan Z, et al. The efficacy of periarticular multimodal drug injection for postoperative pain management in total knee or hip arthroplasty. J Arthroplasty 2013;28:1882-7. 
39. Memtsoudis SG, Sun X, Chiu YL, et al. Perioperative comparative effectiveness of anesthetic technique in orthopedic patients. Anesthesiology 2013;118:1046-58.

40. Pugely AJ, Sun X, Chiu YL, et al. Differences in short-term complications between spinal and general anesthesia for primary total knee arthroplasty. $J$ Bone Joint Surg Am 2013;95:193-9.

41. Gehling M, Tryba M. Risks and side-effects of intrathecal morphine combined with spinal anaesthesia: a meta-analysis. Anaesthesia 2009;64:643-51.

42. Adie S, Kwan A, Naylor JM, et al. Cryotherapy following total knee replacement. Cochrane Database Syst Rev 2012;(9):CD007911.

43. Ni SH, Jiang WT, Guo L, et al. Cryotherapy on postoperative rehabilitation of joint arthroplasty. Knee Surg Sports Traumatol Arthrosc 2015;23:3354-61.

44. Zhang W, Li N, Chen S, et al. The effects of a tourniquet used in total knee arthroplasty: a meta-analysis. J Orthop Surg Res 2014;9:13.

45. Abdel-Salam A, Eyres KS. Effects of tourniquet during total knee arthroplasty. A prospective randomised study. J Bone Joint Surg Br 1995;77:250-3.

46. Ejaz A, Laursen AC, Kappel A, et al. Faster recovery without the use of a tourniquet in total knee arthroplasty. Acta Orthop 2014;85:422-6.

47. Liu D, Graham D, Gillies K, et al. Effects of tourniquet use on quadriceps function and pain in total knee arthroplasty. Knee Surg Relat Res 2014;26:207-13.

48. Dennis DA, Kittelson AJ, Yang CC, et al. Does tourniquet use in TKA affect recovery of lower extremity strength and function? A randomized trial. Clin Orthop Relat Res 2016;474:69-77.

49. Paul JE, Arya A, Hurlburt L, et al. Femoral nerve block improves analgesia outcomes after total knee arthroplasty: a meta-analysis of randomized controlled trials. Anesthesiology 2010;113:1144-62.

50. Ilfeld BM, Duke KB, Donohue MC. The association between lower extremity continuous peripheral nerve blocks and patient falls after knee and hip arthroplasty. Anesth Analg 2010;111:1552-4.

51. Muraskin SI, Conrad B, Zheng N, et al. Falls associated with lower-extremitynerve blocks: a pilot investigation of mechanisms. Reg Anesth Pain Med 2007; 32:67-72.

52. Jaeger P, Nielsen ZJ, Henningsen MH, et al. Adductor canal block versus femoral nerve block and quadriceps strength: a randomized, double-blind, placebocontrolled, crossover study in healthy volunteers. Anesthesiology 2013;118:409-15.

53. Kuang MJ, Ma JX, Fu L, et al. Is adductor canal block better than femoral nerve block in primary total knee arthroplasty? A GRADE analysis of the evidence through a systematic review and meta-analysis. J Arthroplasty 2017;32:3238-48.e3.

54. Goytizolo EA, Lin Y, Kim DH, et al. Addition of adductor canal block to periarticular injection for total knee replacement: a randomized trial. J Bone Joint Surg Am 2019;101:812-20.

55. Lovald ST, Ong KL, Malkani AL, et al. Complications, mortality, and costs for outpatient and short-stay total knee arthroplasty patients in comparison to standard-stay patients. J Arthroplasty 2014;29:510-5.

56. Yang H, Dervin G, Madden S, et al. Postoperative home monitoring after joint replacement: feasibility study. JMIR Perioper Med 2018;1:e10168.

57. Yang H, Dervin G, Madden S, et al. Postoperative home monitoring after joint replacement: retrospective outcome study comparing cases with matched historical controls. JMIR Perioper Med 2018;1:e10169.
58. Gauthier-Kwan OY, Dobransky JS, Dervin GF. Quality of recovery, postdischarge hospital utilization, and 2-year functional outcomes after an outpatient total knee arthroplasty program. J Arthroplasty 2018;33:2159-64.e1.

59. Weiser MC, Kim KY, Anoushiravani AA, et al. Outpatient total hip arthroplasty has minimal short-term complications with the use of institutional protocols. $J$ Arthroplasty 2018;33:3502-7.

60. Cram P, Lu X, Kates SL, et al. Total knee arthroplasty volume, utilization, and outcomes among Medicare beneficiaries, 1991-2010. JAMA 2012;308:1227-36.

61. Bozic KJ, Grosso LM, Lin Z, et al. Variation in hospital-level risk-standardized complication rates following elective primary total hip and knee arthroplasty. J Bone Joint Surg Am 2014;96:640-7.

62. Edelstein Al, Kwasny MJ, Suleiman LI, et al. Can the American College of Surgeons risk calculator predict 30-day complications after knee and hip arthroplasty? J Arthroplasty 2015;30(Suppl 9):5-10.

63. Nelson SJ, Webb ML, Lukasiewicz AM, et al. Is outpatient total hip arthroplasty safe? J Arthroplasty 2017;32:1439-42.

64. Danoff JR, Goel R, Sutton R, et al. How much pain is significant? Defining the minimal clinically important difference for the Visual Analog Scale for Pain after total joint Arthroplasty. J Arthroplasty 2018;33:S71-S75.e2.

65. Churchill L, Pollock M, Lebedeva Y, et al. Optimizing outpatient total hip arthroplasty: perspectives of key stakeholders. Can J Surg 2018;61:370-6.

66. Beaulé PE, Roffey DM, Poitras S. Continuous quality improvement in orthopedic surgery: changes and implications with health system funding reform. Can J Surg 2016;59:149-50.

67. Bertin KC. Minimally invasive outpatient total hip arthroplasty: a financial analysis. Clin Orthop Relat Res 2005;June:154-63.

68. Huang A, Ryu JJ, Dervin G. Cost savings of outpatient versus standard inpatient total knee arthroplasty. Can J Surg 2017;60:57-62.

69. Hip and knee replacements in Canada: Canadian Joint Replacement Registry annual report, 2016-2017. Ottawa: Canadian Institute for Health Information; 2017.

70. Gillis ME, Dobransky J, Dervin GF. Defining growth potential and barriers to same day discharge total knee arthroplasty. Int Orthop 2019;43:1387-93.

71. Bozic KJ, Ward L, Vail TP, et al. Bundled payments in total joint arthroplasty: targeting opportunities for quality improvement and cost reduction. Clin Orthop Relat Res 2014;472:188-93.

72. Duchman KR, Gao Y, Pugely AJ, et al. The effect of smoking on short-term complications following total hip and knee arthroplasty. J Bone Joint Surg Am 2015; 97:1049-58.

73. George DA, Drago L, Scarponi S, et al. Predicting lower limb periprosthetic joint infections: a review of risk factors and their classification. World J Orthop 2017;8:400-11.

74. Bohl DD, Shen MR, Kayupov E, et al. Hypoalbuminemia independently predicts surgical site infection, pneumonia, length of stay, and readmission after total joint arthroplasty. J Arthroplasty 2016;31:15-21.

75. Black CS, Goltz DE, Ryan SP, et al. The role of malnutrition in ninety-day outcomes after total joint arthroplasty. J Arthroplasty 2019;34:2594-2600.

76. Meneghini RM, Ziemba-Davis M. Patient perceptions regarding outpatient hip and knee arthroplasties. J Arthroplasty 2017;32:2701-5.e1.
Competing interests: Paul Beaulé reports receiving royalties from Corin, MicroPort and Medacta; consulting fees from Corin, MicroPort, MatOrtho and Zimmer Biomet; and research support from Zimmer Biomet, outside the submitted work. Dr. Beaulé is a board member of the Canadian Orthopaedic Association and International Society for Hip Arthroscopy. Geoffrey F. Dervin reports receiving personal fees from MicroPort Orthopaedics, outside the submitted work. No other competing interests were declared.

This article has been peer reviewed.
Affiliation: Division of Orthopaedic Surgery, Department of Surgery, University of Ottawa, The Ottawa Hospital, Ottawa, Ont.

Contributors: All of the authors contributed to the conception and design of the work, and the acquisition, analysis and interpretation of data. All of the authors drafted the manuscript, revised it critically for important intellectual content, gave final approval of the version to be published and agreed to be accountable for all aspects of the work.

Correspondence to: Paul Beaulé, pbeaule@toh.ca 